\title{
Índices Climáticos Associados a Variabilidade Interanual da Produtividade de Arroz no Rio Grande do Sul
}

\author{
Diogo Alessandro Arsego ${ }^{1}$ (D), Simone Erotildes Teleginski Ferraz ${ }^{2}$, Nereu Augusto Streck ${ }^{3}$, \\ Andréa de Oliveira Cardoso ${ }^{4}$, Alencar Zanon Junior ${ }^{3}$, Jossana Ceolin Cera ${ }^{5}$ \\ ${ }^{1}$ Centro de Previsão de Tempo e Estudos Climáticos, Instituto Nacional de Pesquisas Espaciais, \\ Cachoeira Paulista, SP, Brasil. \\ ${ }^{2}$ Departamento de Física, Universidade Federal de Santa Maria, Santa Maria, RS, Brasil. \\ ${ }^{3}$ Departamento de Fitotecnia, Universidade Federal de Santa Maria, Santa Maria, RS, Brasil. \\ ${ }^{4}$ Universidade Federal do ABC, Santo André, SP, Brasil. \\ ${ }^{5}$ Instituto Rio Grandense do Arroz, Porto Alegre, RS, Brasil.
}

Recebido em: 18 de Fevereiro de 2018 - Aceito em: 31 de Outubro de 2018

\begin{abstract}
Resumo
O objetivo desta pesquisa é destacar padrões climáticos que exerçam influência na variabilidade interanual da produtividade de arroz no Rio Grande do Sul (RS). Séries de produtividade de arroz de 47 municípios foram separadas em três grupos de acordo com sua produtividade (baixa, média e alta) baseado na análise de agrupamento. Correlações defasadas entre indicadores climáticos associados a padrões de teleconexão e a produtividade média de arroz de cada grupo evidenciaram a importância de cada índice para a variabilidade da produtividade da cultura no RS. Dentre os padrões de teleconexão, os que mais apresentaram correlações significativas e persistentes com a produtividade de arroz foram a Oscilação Quase Bianual (negativa), Oscilação Decadal do Pacífico (negativa), componente atmosférica (positiva) e oceânica (negativa) do fenômeno El Niño Oscilação Sul, além de anomalias de Temperatura da Superfície do Mar no Oceano Atlântico, entre $20^{\circ} \mathrm{S}-30^{\circ} \mathrm{S}$ e $20^{\circ} \mathrm{O}-40^{\circ} \mathrm{O}$, (correlação negativa). A influência de alguns dos indicadores climáticos apresentados neste estudo ainda não havia sido discutida do ponto de vista agrícola e os resultados apresentados podem fornecer informações cruciais na tomada de decisões e na previsão de safra de arroz no Rio Grande do Sul.
\end{abstract}

Keywords: Oryza sativa, teleconnectionpattern, interanual variability.

\section{Climatic Index Associated with the Interannual Variability of Rice Yield in Rio Grande do Sul}

\begin{abstract}
The main of this work is to highlight climatic patterns that influence the interannual variability of rice yield in Rio Grande do Sul (RS). Series of rice yield data from 47 municipalities were separated into three groups according to their yield (low, medium and high) based on clustering analysis. Lag correlations between climatic indicators associated with teleconnections patterns and mean rice yield of each group evidenced the importance of each index for variability of the yield in RS. The most significant and persistent correlations with rice yield were the Quasi-Biennial Oscillation (negative), the Pacific Decadal Oscillation (negative) an atmospheric (positive) and oceanic (negative) components of the El Niño Southern Oscillation. In addition, Sea Surface Temperature anomalies in the Atlantic Ocean, between $20^{\circ} \mathrm{S}-30^{\circ} \mathrm{S}$ and $20^{\circ} \mathrm{O}-40^{\circ} \mathrm{W}$ (negative correlation) have a significant correlation with the rice yield. The influence of some of the climate indices had not yet been discussed from the agricultural point of view. These results can be a crucial information in decision-making and rice yield forecast in Rio Grande do Sul.
\end{abstract}

Palavras chave: Oryza sativa, padrões de teleconexão, variabilidade interanual.

Autor de correspondência: Diogo Alessandro Arsego, diogo.arsego@gmail.com. 


\section{Introdução}

O agronegócio é um dos principais componentes da economia brasileira e do Rio Grande do Sul (RS). A participação do setor agropecuário no Produto Interno Bruto (PIB) do Brasil representa, aproximadamente, $21 \%$ da produção nacional, sendo cerca de $15 \%$ relacionada à atividade agrícola (CEPEA, 2018). O arroz é um dos alimentos mais importantes para a nutrição humana, sendo a base alimentar de mais de três bilhões de pessoas (SOSBAI, 2016). Estima-se que a produção de arroz necessita crescer, ao menos, $40 \%$ nas próximas três décadas para atender as demandas globais (WMO, 2012). O RS é responsável por, aproximadamente, $70 \%$ da produção nacional (SOSBAI, 2016) e, por isso, a compreensão dos fatores e mecanismos responsáveis pela variabilidade da produção de arroz no Estado torna-se fundamental para a segurança alimentar no Brasil.

De todas as atividades econômicas, a agricultura é a mais dependente das condições meteorológicas durante o ciclo de desenvolvimento das culturas agrícolas, tendo como principais variáveis responsáveis pela variabilidade da produtividade à precipitação, temperatura do ar e radiação solar (Hoogenboom, 2000). Em escala global, cerca de um terço das oscilações na produtividade estão associadas a variabilidade climática (Ray et al., 2015). A ocorrência de baixas temperaturas e de baixa disponibilidade de radiação solar durante as fases críticas da planta são fatores que provocam queda de produtividade nas lavouras de arroz irrigado (Mota, 2000, Heinemann et al., 2009, Santos et al., 2017). Em geral, períodos de baixa precipitação não são limitantes à produção de arroz no Rio Grande do Sul, mas excessos de chuva podem prejudicar o crescimento da planta, por reduzir a disponibilidade da radiação solar global durante o ciclo de desenvolvimento da cultura (Heinemann et al., 2009, Santos et al., 2017).

A precipitação sobre o Sul do Brasil é influenciada por fenômenos de variabilidade climática de diferentes escalas. Por exemplo, na escala intrassazonal a Oscilação de Madden-Julian (MJO) pode contribuir para a configuração de um padrão de precipitação favorável a ocorrência de extremos chuvosos na região, o chamado Modo Sul (Pampuch \& Ferraz, 2012). Além disso, há indícios de uma relação positiva entre a atividade da $\mathrm{MJO}$ e o início do El Niño (Hendon et al., 2007). Em escala global, o principal mecanismo responsável pela variabilidade climática interanual é o fenômeno acoplado El Niño Oscilação Sul (ENOS) (Kayano \& Andreoli, 1998) que possui influência marcante sobre a precipitação no RS (Rao \& Hada, 1990; Grimm et al., 1998). Da mesma forma que impacta no regime de chuva no Rio Grande do Sul, evidências mostram que o ENOS exerce papel importante na produtividade de culturas por meio de alterações nos padrões de clima em algumas regiões (Ferreira, 2006; Zubair, 2012). Para o arroz no RS, em geral, episódios de
El Niño são desfavoráveis a cultura enquanto eventos de La Niña estão associados a elevação da produtividade (Carmona, 2001). Em Pelotas, episódios de La Niña forte estiveram associados a produtividade superior a média observada entre 1982 e 1998 (Mota, 2000).

Além do ENOS, outros padrões de teleconexão influenciam no regime de precipitação no RS. Dentre eles, podem ser citados: a Oscilação Decadal do Pacífico (Mantua et al., 1997 - correlação positiva com a precipitação); Oscilação Antártica (Cavalcanti \& Ambrizzi, 2009 - correlação negativa) e anomalias de Temperatura da Superfície do Mar (TSM) no Oceano Atlântico entre $33^{\circ} \mathrm{S}-43^{\circ} \mathrm{S} /$ $48^{\circ} \mathrm{O}-63^{\circ} \mathrm{O}$ (Cataldi et al., 2010 - correlação negativa) e entre $20^{\circ} \mathrm{S}-30^{\circ} \mathrm{S} / 20^{\circ} \mathrm{O}-40^{\circ} \mathrm{O}$ (Santos \& Diniz, 2014 correlação positiva). Apesar disso, a influência de tais padrões na produtividade agrícola do RS ainda não foi devidamente estudada.

Analisando a relação de diversos índices climáticos e os padrões espaciais das componentes principais de vazão no Brasil, Capozolli et al. (2017) mostraram que há relação significativa entre a vazão da Bacia do Uruguai e os índices climáticos relacionados aos padrões atmosféricos Î́ndice de Oscilação Sul, Oscilação do Atlântico Norte, Anomalia de TSM no Atlântico Tropical Sul, Anomalia de TSM na região do Niño 3.4, Modo Meridional do Atlântico, Índice do Oceano Índico, Padrão do Pacífico/América Norte, Oscilação Multidecadal do Atlântico, Oscilação Decadal do Pacífico e Oscilação Antártica, em diferentes defasagens temporais. Dada a relação direta entre precipitação e vazão e tendo em vista que o regime de precipitação também influencia fortemente a produtividade agrícola, a principal pergunta deste artigo é: "Esta relação também é observada entre a produtividade e os indices climáticos?".

Desta forma, o objetivo do presente estudo foi identificar a influência de índices climáticos associados a diferentes padrões de teleconexões na produtividade de arroz no Rio Grande do Sul. A melhor compreensão do impacto destes índices fornecerá informações importantes para auxiliar no planejamento e condução das lavouras de arroz no Rio Grande do Sul.

\section{Material e Métodos}

Foram utilizados dados de produtividade de arroz dos 497 municípios do RS no período compreendido entre 1990 a 2013 (IBGE, 2017). Séries municipais com dados faltantes e/ou repetidos por três anos consecutivos foram eliminadas. Após o primeiro filtro, foram selecionados os municípios com correlação superior a 0,7 com a produtividade média anual do Estado de forma semelhante à utilizada por Berlato \& Fontana (1999) para destacar os municípios produtores de soja no RS. Desta forma, foram excluídos municípios não produtores e não representativos 
para a cultura de arroz irrigado, restando assim, séries de 47 municípios.

A remoção da tendência tecnológica foi realizada com base na abordagem que considera a produtividade ao longo do período dividido em duas séries: tendência e variação (Wenjiao et al., 2013). Assim, assume-se que a tendência esteja unicamente relacionada com o aumento da tecnologia e a sua remoção usando a Eq. (1) fornece uma série temporal que, em tese, contém apenas variações climáticas.

$$
Y_{c i}=Y_{i}-Y\left(X_{i}\right)-Y\left(X_{f}\right)
$$

sendo que $Y_{c i}$ é a produtividade corrigida para o ano $i ; Y_{i}$ é a produtividade original do ano $i ; Y\left(X_{i}\right)$ é a produtividade estimada para o ano $i$ através do modelo de regressão e $Y$ $\left(X_{f}\right)$ é a produtividade do último ano estimada pela regressão linear.

As séries históricas de produtividade foram distribuídas em grupos homogêneos de alta, média e baixa produtividade tendo como base a distância Euclidiana (Wilks, 2006) e com agrupamento realizado pelo método hierárquico proposto por Ward (1963), no qual a distância entre dois grupos (clusters) é a soma das distâncias ao quadrado. A formação destes grupos permite a obtenção de uma série média de produtividade que represente a variabilidade interanual de cada grupo. Os perfis médios obtidos foram então relacionados a forçantes oceânicos e atmosféricos de forma a obter os indicadores climáticos com maior influência em cada grupo.

A análise da influência dos padrões de teleconexão na variabilidade interanual da produtividade de arroz no Rio Grande do Sul foi estudada com base na análise de correlação entre a série de produtividade média para cada grupo homogêneo e os seguintes indicadores climáticos disponibilizados pela página do "National Oceanic and Atmospheric Administration - NOAA": Anomalia de TSM na região do Niño 3.4 (NINO - (Trenberth, 1997)); Índice de Oscilação Sul (IOS - (Trenberth, 1984)); Oscilação do Atlântico Norte (NAO - (Rogers, 1985)); Padrão do Pacífico / América Norte (PNA - (Wallace et al., 1981)); Oscilação Multidecadal do Atlântico (AMO - (Schelesinger \& Raman-Kutty, 1994)); Anomalia de TSM no Atlântico Tropical Sul (TSA - (Enfield et al., 1999)); Anomalia de TSM no Atlântico Tropical Norte (TNA - (Enfield et al., 1999)); Oscilação Decadal do Pacífico (ODP (Mantua et al., 1997)); Oscilação Quase Bienal (QBO (Angell \& Korshover, 1962)); Modo Meridional do Atlântico (AMM - (Servain, 1991)); Oscilação Ártica (AO (Thompson \& Wallace, 1998)) e Oscilação Antártica (OAA - (Thompson \& Wallace, 2000)).

Além dos indicadores acima listados, foram adicionados outros dois índices: ATSM1 e ATSM2. O índice ATSM1 é referente a região do Atlântico Sul delimitada entre as latitudes de $20^{\circ} \mathrm{S}$ e $30^{\circ} \mathrm{S}$ e as longitudes $20^{\circ} \mathrm{O}$ e $40^{\circ}$ O. Anomalias de Temperatura da Superfície do Mar (TSM) nessa região possuem correlação positiva com a precipitação no Rio Grande do Sul, principalmente entre os meses de outubro e janeiro (Santos \& Diniz, 2014). O índice ATSM2 está relacionado a região oceânica compreendida entre $33^{\circ} \mathrm{S}$ e $43^{\circ} \mathrm{S}$ e $48^{\circ} \mathrm{O}$ e $63^{\circ} \mathrm{O}$. Anomalias positivas (negativas) de TSM na região de encontro entre as Correntes do Brasil e das Malvinas alteram o regime de chuvas e favorecem precipitações abaixo (acima) da média no Sul (Sudeste) (Cataldi et al., 2010).

Estes índices foram criados por meio da normalização dos dados observados da seguinte forma (Eq. (2)):

$$
\frac{\left(x_{\mathrm{ij}}-\overline{x_{i}}\right)}{\sigma_{i}}
$$

sendo $x_{\mathrm{ij}}$ o valor observado no mês $i$ e ano $j$, no caso da TSM o valor corresponde a média da TSM na região de estudo; $\overline{x_{i}}$ a média de todas as observações do mês $i$ e $\sigma_{i} \mathrm{O}$ desvio padrão das observações no mês $i$.

Para analisar a influência de cada índice em cada grupo homogêneo da cultura do arroz, foram realizadas correlações defasadas entre os índices climáticos mensais de cada padrão de teleconexão e a produtividade média anual do arroz dos três grupos. Estabeleceu-se o mês de março como mês referente ao início da colheita com base no calendário médio da cultura delimitado em Klering et al. (2016). Desta forma, indicadores climáticos de abril a dezembro foram relacionados um a um com o valor de produtividade do ano seguinte enquanto indicadores de janeiro a março foram correlacionados com o valor de produtividade do ano correspondente. Assim, a primeira correlação é tomada entre o índice climático referente ao mês de abril do ano anterior e a produtividade do ano subsequente (lag 11), a segunda entre o índice do mês de maio do ano anterior e a produtividade do ano seguinte (lag 10) e assim, sucessivamente, até a última correlação entre o índice do mês de março do ano referente à colheita e o valor de produtividade do mesmo ano (lag0).

A significância estatística foi definida através do teste $\mathrm{t}$ de Student (Fischer \& Yates, 1974) com $n-2$ graus de liberdade, sendo $n$ o número de casos (24 anos de dados) e $t_{0}=\frac{r \sqrt{n-2}}{\sqrt{1-r^{2}}}$. Desta forma, para um nível de confiança de 95\%, o valor limite para a correlação é: $|r|>0,345$.

\section{Resultados e Discussão}

A distribuição dos municípios produtores de arroz em grupos homogêneos evidencia que os municípios do Grupo 1 (G1 - Fig. 1) apresentam a menor produtividade média (Fig. 2) e se distribuem pelas regiões orizícolas da Região Central e das Planícies Interna e Externa a Lagoa dos Patos segundo o Instituto Rio Grandense do Arroz (IRGA). O Grupo 2 (Fig. 1), de produtividade interme- 


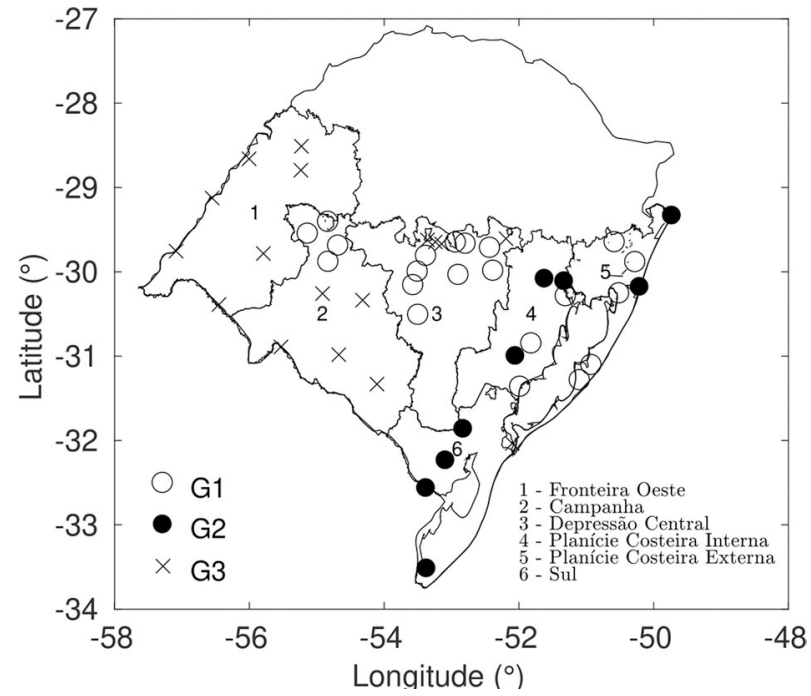

Figura 1 - Mapa das regiões homogêneas de produtividade de arroz no Rio Grande do Sul com a distribuição espacial dos municípios classificados nos Grupos 1 (círculo vazio), 2 (círculo cheio) e 3 (X). Os limites e a numeração referem-se as regiões orizícolas do Estado segundo o IRGA.

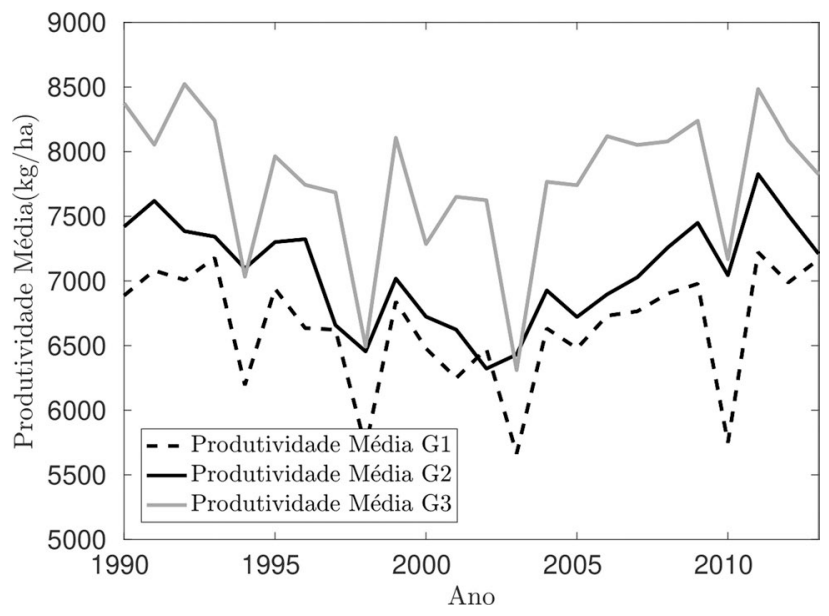

Figura 2 - Perfis de produtividade média anual de arroz durante no período entre 1990 a 2013 para os grupos homogêneos: G1 (linha preta tracejada), G2 (linha contínua preta) e G3 (linha contínua cinza).

diária (Fig. 2), encontra-se, principalmente, no Sul do Estado e, de forma mais isolada, em pontos das Planícies em torno da Lagoa dos Patos. Por fim, o Grupo 3 (Fig. 1), grupo de maior produtividade (Fig. 2), apresenta a maior parte de seus integrantes sobre as regiões da Fronteira Oeste e Campanha gaúcha.

Segundo o zoneamento agroclimático do arroz no RS (EMBRAPA, 2001), a Fronteira Oeste apresenta a maior disponibilidade de radiação solar $\left(23,5 \mathrm{MJ} /\left(\mathrm{m}^{2}\right.\right.$.dia $\left.)\right)$ nas fases críticas (florescimento e enchimento de grãos) do desenvolvimento das lavouras de arroz irrigado e menor risco em relação a temperaturas baixas $\left(24,5^{\circ} \mathrm{C}\right)$ fator que explicaria as maiores produtividades encontradas para o Grupo 3 (Fig. 2). Por outro lado, a Região Central encon- tra-se em uma faixa onde a disponibilidade de radiação solar é menor (22 MJ/( $\mathrm{m}^{2}$.dia $\left.)\right)$ e, por isso, os municípios situados nesta região (Fig. 1 - G1) apresentam menores produtividades médias em comparação com as outras regiões do Estado (Fig. 2). Além disso, lavouras na Fronteira Oeste são semeadas em setembro até a metade de outubro, fazendo com que o florescimento e o enchimento de grãos coincidam com o período de maior disponibilidade de radiação solar maximizando assim, os componentes de produtividade, enquanto as lavouras da Região Central são semeadas no final de outubro e novembro, tendo assim menor potencial de produtividade. Desta forma, o agrupamento baseado nos dados de produtividade é justificado pelas características climáticas e critérios descritos no zoneamento agroclimático da cultura no RS.

As correlações entre os índices climáticos do mês de abril e a produtividade de arroz nos três grupos (Fig. 3), destacam as correlações entre a OAA e o G2 (negativa), QBO e os grupos 1 e 3 (negativa) e as anomalias de TSM na região da ATSM1 e o G3 (positiva). Entre os índices acima citados, se sobressai a QBO com sinal persistente para os meses de maio e junho (Fig. 1) para os grupos 1 e 3 e no mês de julho apenas para o G3 (Fig. 3). Um estudo preliminar de Gava et al. (2017) sugere que a QBO exerça influência na Oscilação Antártica, padrão este que possui impacto na precipitação sobre o RS (Cavalcanti \& Ambrizzi, 2009). Além disso, estudos também mostram uma relação entre o ENOS e a QBO (Lau e Sheu, 1988, Yuan et al., 2014), o que também pode ter contribuído para as correlações encontradas neste estudo. Análises futuras mais aprofundadas poderão auxiliar na melhor compreensão da influência da QBO na variabilidade climática do RS e, mais especificamente, na produtividade de arroz.

A partir do mês de maio (Fig. 3), inicia a influência do ENOS na produtividade de arroz. Em maio, apenas a sua componente atmosférica (IOS) exerce influência (correlação positiva e significativa) sobre os três grupos. O sinal associado ao IOS persiste com correlações significativas até o mês de outubro (Fig. 4), alternando sua intensidade entre os grupos. Tal correlação estaria associada à persistência de um padrão de tempo mais seco durante o período de desenvolvimento da cultura que favorece a elevação da produtividade pela maior disponibilidade de radiação solar. Esta hipótese pode ser confirmada pela correlação negativa entre as anomalias de TSM no Niño 3.4 (Niño) configurada entre junho (Fig. 3) e março (Fig. 5) com pequenas alterações em sua intensidade e significância ao longo dos meses. Os resultados aqui encontrados para o fenômeno ENOS corroboram com estudos anteriores que sinalizaram que eventos de La Niña estão associados ao aumento da produtividade de arroz no sul do Estado (Mota, 2000). A ligeira diferença entre as correlações dos grupos poderia ser explicada, em parte, pela menor correlação entre as TSMs do Pacífico Equatorial e a precipitação no sul do Estado (G2) durante os 

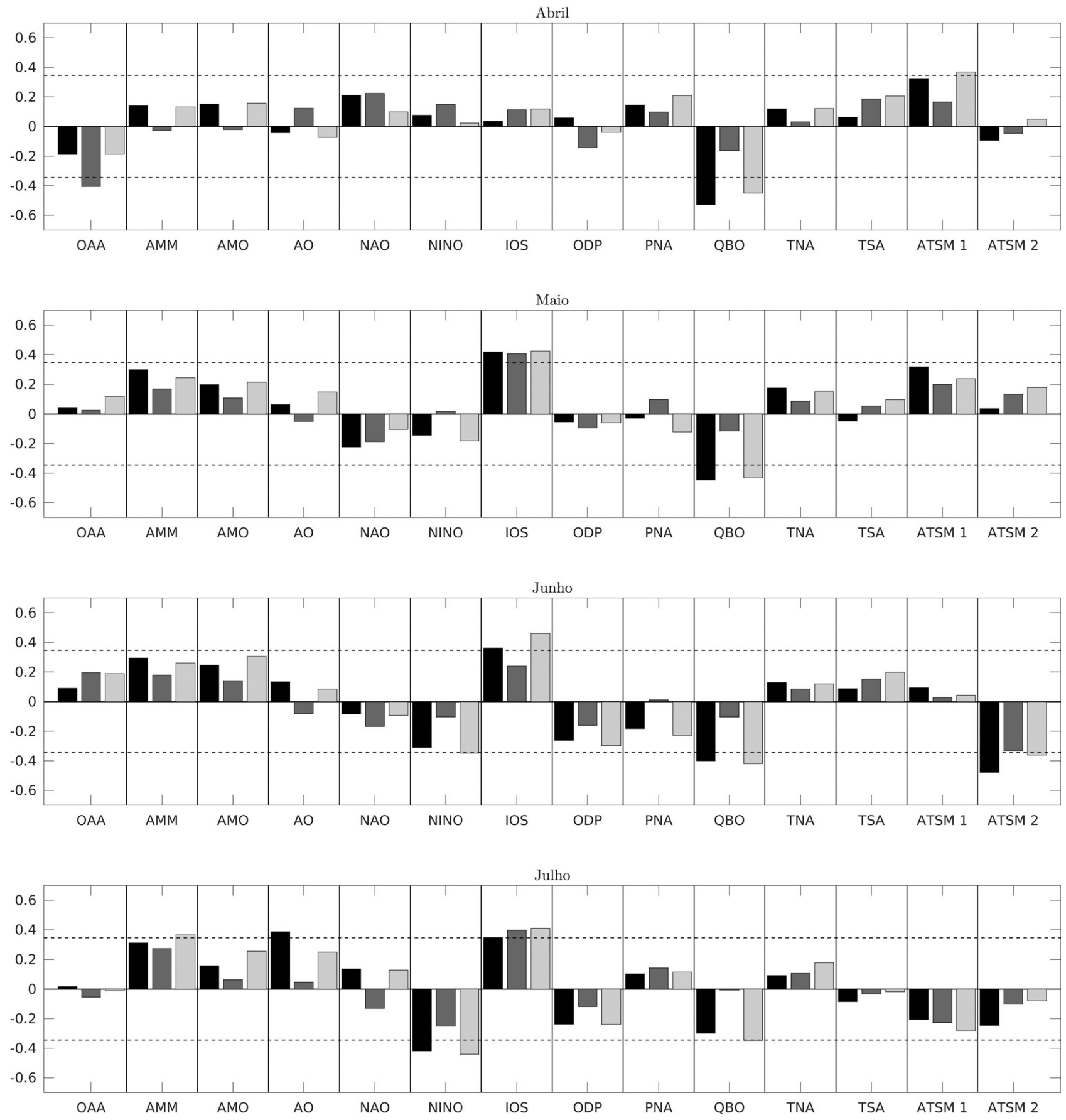

Figura 3 - Correlação entre os indicadores climáticos de abril a julho e a produtividade de arroz para os três grupos homogêneos: G1 (preto), G2 (cinza escuro) e G3 (cinza claro). As linhas horizontais pontilhadas delimitam o nível de significância de $95 \%$. Os índices utilizados foram: Oscilação Antártica (OAA), Modo Meridional do Atlântico (AMM), Oscilação Multidecadal do Atlântico (AMO), Oscilação Ártica (AO), Oscilação do Atlântico Norte (NAO), Anomalia de TSM na região do Niño 3.4 (NINO), Índice de Oscilação Sul (IOS), Oscilação Decadal do Pacífico (ODP), Padrão do Pacífico / América Norte (PNA), Oscilação Quase Bienal (QBO), Anomalia de TSM no Atlântico Tropical Norte (TNA), Anomalia de TSM no Atlântico Tropical Sul (TSA), e anomalias de TSM entre $20^{\circ} \mathrm{S}-30^{\circ} \mathrm{S}$ e $20^{\circ} \mathrm{O}-40^{\circ} \mathrm{O}$ (ATSM1) e entre $33^{\circ} \mathrm{S}-43^{\circ} \mathrm{S}$ e $48^{\circ} \mathrm{O}-63^{\circ} \mathrm{O}$ (ATSM2).

meses de janeiro a março em comparação com os grupos 1 e, principalmente, G3 (Lopes et al., 2007).

Para os indicadores climáticos do mês de junho (Fig. 3), nota-se a influência das anomalias de TSM referentes ao índice ATSM2 com o G1 e com o G3. Esta re- gião do Atlântico sul está negativamente correlacionada com a precipitação no RS (Cataldi et al., 2010). Este sinal negativo no mês de junho e a não persistência para os meses seguintes poderia indicar que anomalias positivas (negativas) nesta região estariam associadas a uma dimi- 

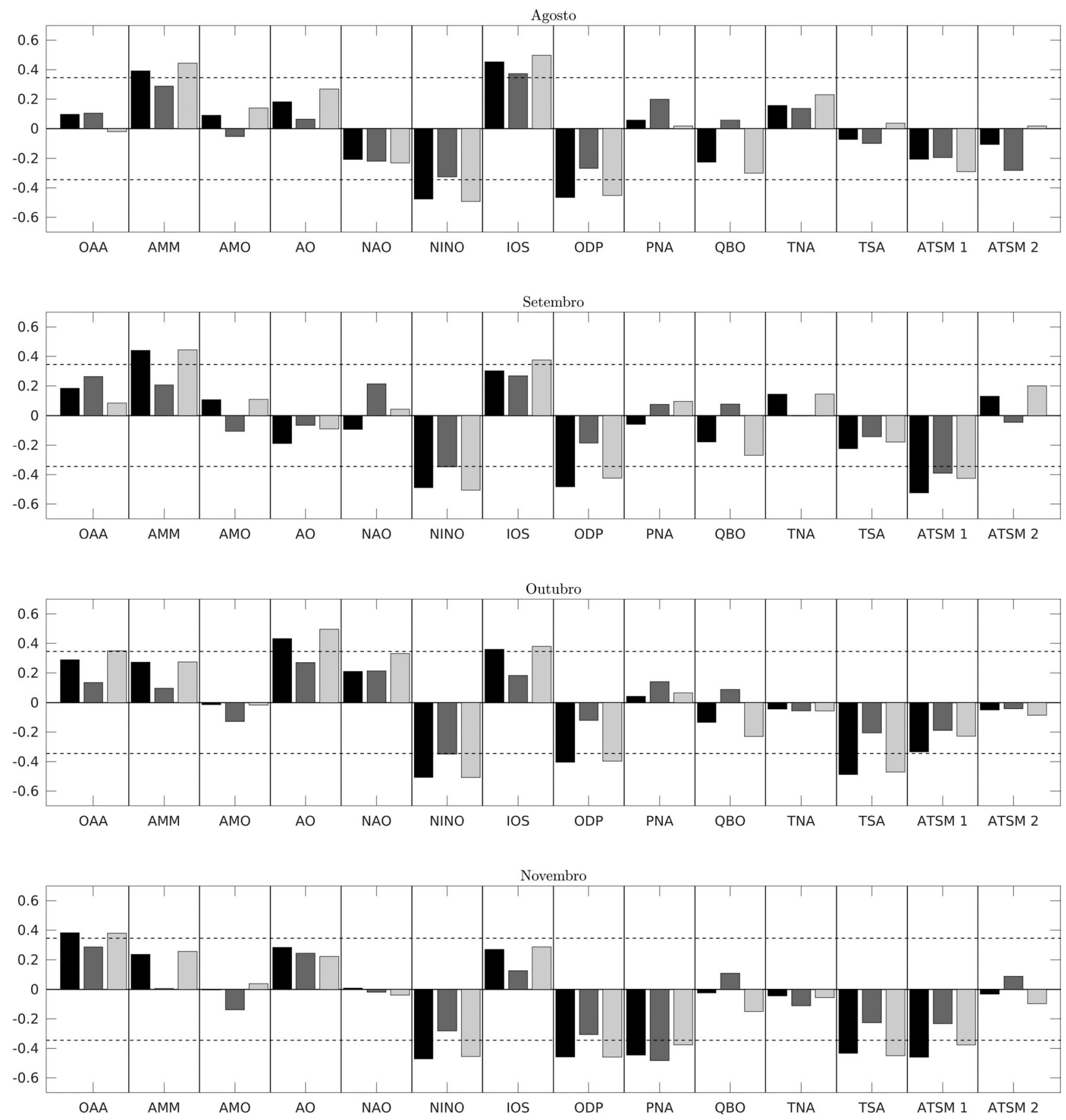

Figura 4 - Correlação entre os indicadores climáticos de agosto a novembro e a produtividade de arroz para os três grupos homogêneos: G1 (preto), G2 (cinza escuro) e G3 (cinza claro). As linhas horizontais pontilhadas delimitam o nível de significância de 95\%. Os índices utilizados foram: Oscilação Antártica (OAA), Modo Meridional do Atlântico (AMM), Oscilação Multidecadal do Atlântico (AMO), Oscilação Ártica (AO), Oscilação do Atlântico Norte (NAO), Anomalia de TSM na região do Niño 3.4 (NINO), Índice de Oscilação Sul (IOS), Oscilação Decadal do Pacífico (ODP), Padrão do Pacífico / América Norte (PNA), Oscilação Quase Bienal (QBO), Anomalia de TSM no Atlântico Tropical Norte (TNA), Anomalia de TSM no Atlântico Tropical Sul (TSA), e anomalias de TSM entre $20^{\circ} \mathrm{S}-30^{\circ} \mathrm{S}$ e $20^{\circ} \mathrm{O}-40^{\circ} \mathrm{O}$ (ATSM1) e entre $33^{\circ} \mathrm{S}-43^{\circ} \mathrm{S}$ e $48^{\circ} \mathrm{O}-63^{\circ} \mathrm{O}$ (ATSM2).

nuição (elevação) da precipitação no período de enchimento dos reservatórios e, por este motivo, resultariam em queda (aumento) da produtividade do arroz.

Entre julho e agosto (Figs. 3 e 4), indicadores climáticos associados a padrões de teleconexão com maior influência sobre o Hemisfério Norte passam a exercer influência sugerindo assim, a hipótese de influências inter-hemisféricas. A AO possui correlação positiva com a produtividade do G1 para julho (Fig. 3). Após um período com ausência de sinal significativo, a AO volta a 

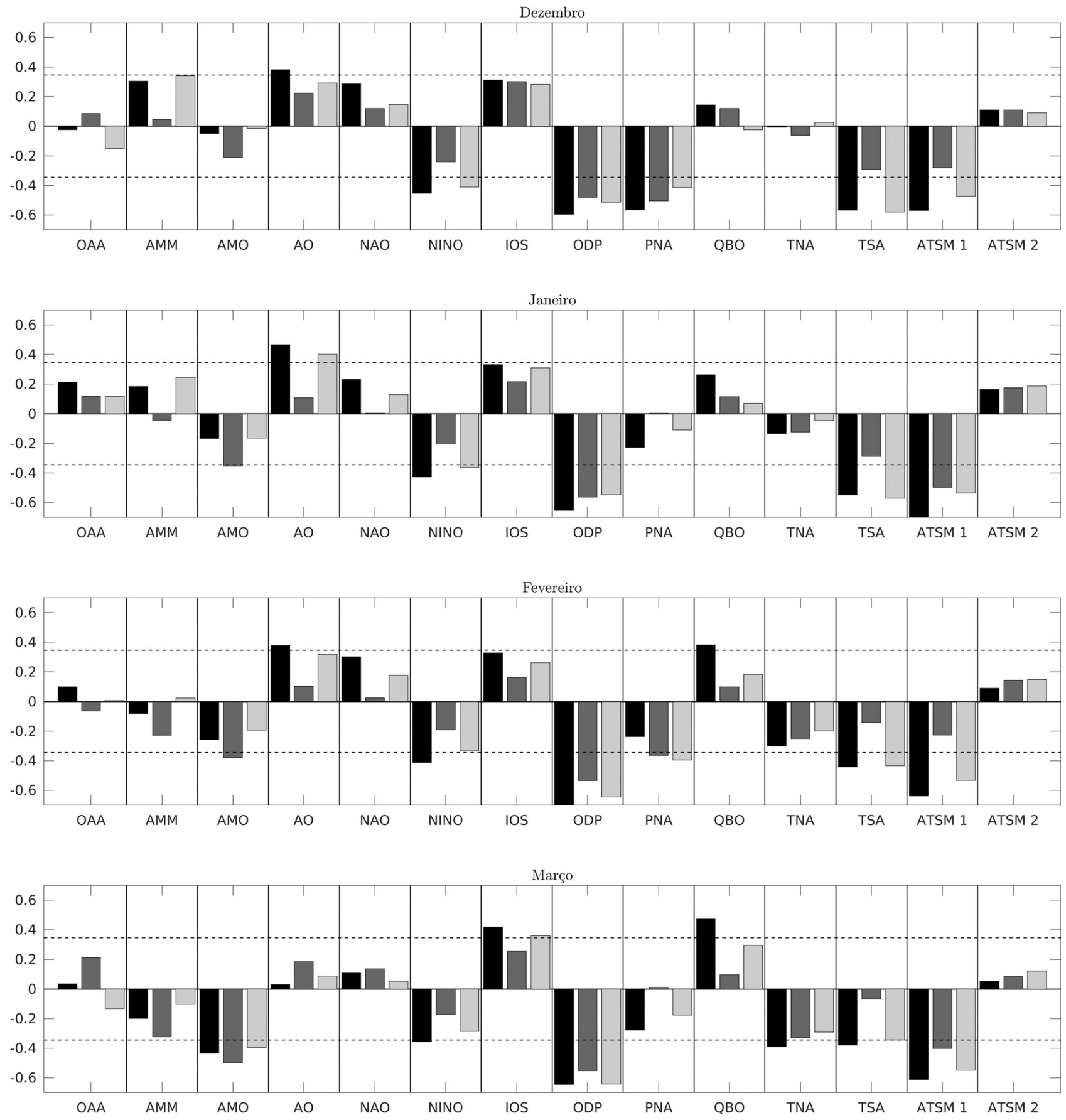

Figura 5 - Correlação entre os indicadores climáticos de dezembro a março e a produtividade de arroz para os três grupos homogêneos: G1 (preto), G2 (cinza escuro) e G3 (cinza claro). As linhas horizontais pontilhadas delimitam o nível de significância de 95\%. Os índices utilizados foram: Oscilação Antártica (OAA), Modo Meridional do Atlântico (AMM), Oscilação Multidecadal do Atlântico (AMO), Oscilação Ártica (AO), Oscilação do Atlântico Norte (NAO), Anomalia de TSM na região do Niño 3.4 (NINO), Índice de Oscilação Sul (IOS), Oscilação Decadal do Pacífico (ODP), Padrão do Pacífico / América Norte (PNA), Oscilação Quase Bienal (QBO), Anomalia de TSM no Atlântico Tropical Norte (TNA), Anomalia de TSM no Atlântico Tropical Sul (TSA), e anomalias de TSM entre $20^{\circ} \mathrm{S}-30^{\circ} \mathrm{S}$ e $20^{\circ} \mathrm{O}-40^{\circ} \mathrm{O}$ (ATSM1) e entre $33^{\circ} \mathrm{S}-43^{\circ} \mathrm{S}$ e $48^{\circ} \mathrm{O}-63^{\circ} \mathrm{O}$ (ATSM2).

apresentar correlação com seu índice do mês de outubro (positiva para G1 e G3 - Fig. 4), dezembro (positiva para o G1 - Fig. 5), janeiro (positiva para G1 e G3 - Fig. 5) e fevereiro (positiva para G1 - Fig. 5). Embora os mecanismos físicos responsáveis por esta ligação ainda neces- sitem ser melhores compreendidos, a persistência de correlações significativas da $\mathrm{AO}$ com a produtividade de arroz no RS indica que o monitoramento deste índice pode ser útil em futuros planejamentos de safra agrícola no Estado. 
A AMM apresenta correlação positiva com o G3 no mês de julho (Fig. 3) e com o G1 e G3 em agosto e setembro (Fig. 4). A partir do mês de agosto (Fig. 4) a ODP começa a apresentar significativo e persistente sinal sobre a produtividade de arroz, quando também se destaca o índice oceânico do Niño. Para o G1 e G3 a correlação é significativa entre os meses de agosto a março (Figs. 4 e 5). Para o G2, a correlação passa a ser significativa a partir do mês de dezembro e persiste até o mês de março (Fig. 5). Quando ODP e ENOS estão em fase, os episódios de El Niño e La Niña podem ser mais intensos e frequentes (Kayano \& Andreoli, 1998) e, por este motivo, o sinal negativo da ODP com a produtividade de arroz já era esperado. Streck et al. (2009) já haviam demonstrado a correlação positiva entre a ODP e a precipitação na região central do RS (G1) nas escalas interanual, sazonal e mensal.

A região do Atlântico delimitada pelos indicadores referentes ao índice ATSM1 passa a apresentar correlação negativa a partir do mês de setembro (Fig. 4). O sinal negativo e sua persistência durante o desenvolvimento da cultura ocorre por conta de sua influência direta na precipitação sobre o Estado (Santos \& Diniz, 2014). Entre outubro e março (Figs. 4 e 5), nota-se a influência da TSA com correlação negativa com a produtividade de arroz nos grupos 1 e 3. O PNA exerce influência negativa para os três grupos em novembro e dezembro e para os grupos $2 \mathrm{e}$ 3 em fevereiro (Figs. 4 e 5).

A OAA apresenta correlação positiva com o $\mathrm{G} 3 \mathrm{em}$ outubro e com os grupos 1 e 3 no mês de novembro (Fig. 4). Este resultado era esperado em virtude de sua relação direta com o avanço de sistemas frontais sobre o RS e, consequentemente, influência na precipitação do Estado (Cavalcanti \& Ambrizzi, 2009). Fases positivas (negativas) da OAA estão associadas a menor (maior) atividade frontal sobre o Estado e, consequentemente, diminuição (elevação) da precipitação.

Por fim, nos meses de janeiro a março (Fig. 5), notase a influência da AMO (correlação negativa) com o G2 para todo o período e com os grupos 1 e 3 apenas para março. A TNA apresenta correlação significativa (negativa) apenas no mês de março com o G1 (Fig. 5).

Muitos dos resultados aqui apresentados são inéditos do ponto de vista agrometeorológico tendo em vista que, apenas as influências do ENOS na variabilidade da produtividade do arroz no RS foram estudadas anteriormente. A relação entre produtividade e índices associados a padrões de teleconexão corrobora com estudos prévios e serve para ressaltar novos índices cuja influência no regime de chuva e temperatura no RS e sua resposta na produtividade de arroz ainda precisa ser melhor entendida. Ainda assim, a principal contribuição deste trabalho é a demonstração da forte influência que outros padrões de teleconexão apresentam na variabilidade da produtividade de arroz no RS.
O principal resultado aqui apresentado são as amplas e persistentes correlações da QBO com a produtividade nos Grupos 1 e 3. Tal defasagem (4 a 6 meses antes do plantio) fornece informações cruciais para planejamento da safra de arroz no RS. A utilização de tais índices pode sinalizar um avanço nas estimativas de produtividade e previsão de safra, bem como auxiliar no planejamento e tomada de decisão de práticas de manejo nas lavouras de arroz no RS. Além disso, os índices aqui destacados com maiores correlações poderão, futuramente, servir de base de um modelo estatístico de previsão de safra de arroz no Rio Grande do Sul.

Em relação à pergunta inicial deste artigo, nem todos índices relacionados a padrões climáticos aqui estudados (e que apresentaram uma relação com a vazão), apresentaram correlação com a produtividade. Isto pode estar relacionado ao fato de a produtividade do IBGE ser um único valor anual, ou seja, este valor possui influência das condições climáticas em todas fases da cultura, na qual poderia ser resolvido acompanhando-se a cultura durante todo o seu ciclo.

\section{Conclusões}

O agrupamento dos municípios baseado em dados de produtividade de arroz demonstrou que a Fronteira Oeste e Campanha são as regiões com maiores produtividades de arroz irrigado enquanto a Região Central é a que apresenta a menor produtividade média.

O fenômeno acoplado El Niño Oscilação Sul apresenta correlações positivas (negativas) significativas entre sua componente oceânica (atmosférica) e a produtividade de arroz irrigado no RS.

A Oscilação Quase Bienal (QBO) apresenta persistentes correlações negativas com a produtividade de arroz nos grupos 1 e 3, especialmente de 4 a 6 meses antes do plantio. Isto sugere que o monitoramento dos índices relacionados a esta oscilação fornecem informações cruciais para planejamento da safra de arroz no RS.

A Oscilação Decadal do Pacífico (ODP) apresenta correlações negativas com a produtividade de arroz no Rio Grande do Sul com 2 meses de antecedência em relação ao período médio da semeadura do arroz (outubro). Isso faz dela um indicador preditivo para o período de pré-semeadura.

As anomalias de Temperatura da Superfície do Mar entre $20^{\circ} \mathrm{S}-30^{\circ} \mathrm{S}$ e $20^{\circ} \mathrm{O}-40^{\circ} \mathrm{O}$ destacaram-se com correlações negativas com a produtividade de arroz no Rio Grande do Sul durante o período de desenvolvimento da cultura sendo a análise desta variável recomendada para estimativas de produtividade durante esta fase. 


\section{References}

ANGELL, J.K.; KORSHOVER, J. The biennial wind and temperature oscillations of the equatorial stratosphere and their possible extension to higher latitudes. Monthly Weather Review, v. 90, n. 4, p. 127-132, 1962.

BERLATO, M.A.; FONTANA, D.C. Variabilidade interanual da precipitação pluvial e rendimento da soja no Rio Grande do Sul. Revista Brasileira de Agrometeorologia, v. 7, n. 1, p. 119-125, 1999.

CAPOZZOLI, C.R.; CARDOSO, A. de O.; FERRAZ, S.E.T. Padrões de Variabilidade na Vazão de Rios nas Principais Bacias Brasileiras e Associação com Índices Climáticos. Revista Brasileira de Meteorologia, v. 32, n. 2, p. 243254, 2017.

CARMONA, L. de C. Efeitos associados aos fenômenos El Niño e La Niña no rendimento do arroz irrigado no Estado do Rio Grande do Sul. 2001. 77 f. Dissertação (Mestrado em Fitotecnia) - Universidade Federal do Rio Grande do Sul, Porto Alegre.

CATALDI, M.; ASSAD, L.P. de F.; JUNIOR, A.R.T.; ALVEZ, J.L.D. Estudo da influência das anomalias da TSM do Atlântico Sul Extratropical na Região da Confluência Brasil Malvinas no regime hidrometeorológico de verão no Sul e Sudeste do Brasil. Revista Brasileira de Meteorologia, v. 25, n. 4, p. 513-524, 2010.

CAVALCANTI, I.F.A.; AMBRIZZI, T. Teleconexões e suas influências no brasil. In: CAVALCANTI, I. F. de A. et al. (Ed.). Tempo e Clima no Brasil. Oficina de Textos, São Paulo, 2009. p. 317-336.

CEPEA. PIB Agro CEPEA-USP/CNA. 2018. Disponível em: $<$ http://cepea.esalq.usp.br/br/pib-do-agronegocio-brasi leiro.aspx> . Acesso em: Setembro de 2018.

EMBRAPA. Macrozoneamento Climático para o Arroz Irrigado no Rio Grande do Sul. 2005. Disponível: <http:// agromet.cpact.embrapa.br/zoneamento/macro_arroz/con sulta.php>. Acesso em: Abril 2017.

ENFIELD, D.B.; MESTAS-NUÑEZ, A.M.; MAYER, D.A.; CID-SERRANO, L. How ubiquitous is the dipole relationship in tropical atlantic sea surface temperatures? Journal of Geophysical Research, v. 104, n. C4, p. 7841-7848, 1999.

FERREIRA, D.B. Relação entre a variabilidade da precipitação e a produtividade agrícola de soja e milho nas Regiões Sul e Sudeste do Brasil. 2006. 125 f. Dissertação (Mestrado em Meteorologia) - Instituto Nacional de Pesquisas Espaciais, São José dos Campos.

FISHER, R.A.; YATES, F. Statistical Tables for Biological, Agricultural and Medicinal Research. 6. Ed. Longman, London, 1974. 146 p.

GAVA, M.L.L.M; VASCONCELLOS, F.C.; SANGIOLO, C.A. Um estudo sobre a possível relação entre a Oscilação Quase-Bienial e a Oscilação Antártica. In: Anais do VII SIMPÓSIO INTERNACIONAL DE CLIMATOLOGIA, 2017, Petrópolis (RJ). Sociedade Brasileira de Meteorologia, 2017.

GRIMM, A.M.; FERRAZ, S.E.T.; GOMES, J. Precipitation Anomalies in Southern Brazil Associated with El Niño and La Niña Events. Journal of Climatology, v. 11, p. 28632880, 1998.
HEINEMANN, A.B.; STONE, L.F.; SILVA, S.C. da.Arroz. In: Agrometeorologia dos Cultivos. [S.1.: s.n.], 2009. p. 6380.

HENDON, H.H.; WHEELER, M.C.; ZHANG, C. Seasonal dependence of the MJO-ENSO relationship. Journal of Climate, v. 20, n. 3, p. 531-543, 2007.

HOOGENBOOM, G. Contribution of agrometeorology to the simulation of crop production and its applications. Agricultural and Forest Meteorology, v. 103, p. 137-157, 2000.

IBGE. Produção Agrícola Municipal. 2017. Disponível em: $<$ https://sidra.ibge.gov.br/Tabela/1612>. Acesso em: Abril 2017.

KAYANO, M.T.; ANDREOLI, R.T. Relations of South American summer rainfall interannual variations with the Pacific Decadal Oscillation. Journal of Climate, v. 11, p. 28632880, 1998.

KLERING, E.V.; FONTANA, D.C.; ROGLIO, V.S.; ALVES, R. de C.M.; BERLATO, M. A. Modelo agrometeorológicoespectral para estimativa da produtividade de grãos de arroz irrigado no rio grande do sul. Bragantia, v. 75 , n. 2 , p. 247-256, 2016.

LAU, K.M.; SHEU, P.J. Annual Cycle, Quasi-Biennial Oscillation, and Southern Oscillation in Global Precipitation. Journal of Geophysical Research, v. 93, n. D9, p. 975988, 1988.

LOPES, F.Z.; DINIZ, G.B.; MARQUES, J.R. Relação entre o multivariateenso index (mei) e a TSM das regiões dos niños com a precipitação em regiões homogêneas do estado do Rio Grande do Sul. Anuário do Instituto de Geociências, v. 30, p. 11-22, 2007.

MANTUA, N.J.; HARE, S.R.; ZHANG, Y. WALLACE, J.M.; FRANCIS, R.C.A Pacific Interdecadal Climate Oscillation with Impacts on Salmon Production. Bulletin of the American Meteorological Society, v. 78, n. 6, p. 10691079, 1997.

MOTA, F.S. da.Influência dos fenômenos El Niño e La Niña sobre o rendimento do arroz irrigado na região de Pelotas (RS). Revista Brasileira de Meteorologia, v. 15, n. 2, p. 21-24, 2000.

PAMPUCH, L.A.; FERRAZ, S.E.T. Investigação do Modo Sul em dados de precipitação no período de 1982 a 2006 no estado do Rio Grande do Sul. Revista Brasileira de Meteorologia, v. 27, n. 1, p. 107-116, 2012.

RAO, G.V.; HADA, K. Characteristics of rainfall over Brazil annual variations and connections with the Southern Oscillation. Theoretical and Applied Climatology, v. 42, p. 8191, 1990.

RAY, D.K.; GERBER, J.S.; MACDONALD, G.K.; WEST, P.C. Climate variation explains a third of global crop yield variability. Nature Communications, v. 6, p. 1-9, 2015.

ROGERS, J.C. Atmospheric circulation changes associated with the warming over the northern north atlantic in the $1920 \mathrm{~s}$. Journal of Climate, v. 24, n. 12, p. 1303-1310, 1985.

SANTOS, E.B.; DINIZ, G.B. Oceanic indices and their relations with the monthly precipitation in Rio Grande do Sul state, Brazil. Revista Brasileira de Geofísica, v. 32, p. 371-381, 2014.

SANTOS, M.P.; ZANON, A.J.; CUADRA, S.V.; STEINMETZ, S.; CASTRO, J.R.; HEINEMANN, A. B. Yield and mor- 
phophysiological indices of irrigated rice genotypes in contrasting ecosystems. Pesquisa Agropecuaria Tropical (Online), v. 47, p. 253-264, 2017.

SCHLESINGER, M.E.; RAMANKUTTY, N. An oscillation in the global climate system of period 65-70 years. Nature, v. 367, n. 6465, p. 723-726, 1994.

SERVAIN, J. Simple climatic indices for the tropical atlantic ocean and some applications. Journal of Geophysical Research, v. 96, n. C8, p. 15137-15146, 1991.

SOSBAI [Sociedade Sul Brasileira de Arroz Irrigado]. 2016. Arroz Irrigado: recomendações técnicas da pesquisa para o Sul do Brasil. Bento Gonçalves, 2016. 200 p.

STRECK, N.A.; BURIOL, G.A.; HELDWEIN, A.B.; GABRIEL, L.F.; de PAULA, G.M. Associação da variabilidade da precipitação pluvial em Santa Maria com a Oscilação Decadal do Pacífico. Pesquisa Agropecuária Brasileira, v. 44, n. 12, p. 1553-1561, 2009.

THOMPSON, D.W.J.; WALLACE, J. M.The arctic oscillation signature in the wintertime geopotential height and temperature fields. Geophysical Research Letters, v. 25, p. $1297-1300,1998$.

THOMPSON, D.W.J.; WALLACE, J.M. Annular modes in the extratropical circulation.part1: Month-to-month variability. Journal of Climate, v. 13, p. 1000-1016, 2000.

TRENBERTH, E.K. Signal versus noise in the southern oscillation. Monthly Weather Review, v. 112, n. 2, p. 326-332, 1984.

TRENBERTH, E.K. The definition of El Niño. Bulletin of American Meteorological Society, v. 28, n. 12, p. 27712777, 1997.
WALLACE, J.M.; FULU,T.; ZHAO, Z. Teleconnections in the geopotential height field during the north hemisphere winter. Monthly Weather Review, v. 109, n. 4, p. 784-812, 1981.

WARD, J.H. Hierarchical grouping to optimize an objective function. Journal of the American Statistical Association, v. 58, n. 301, p. 236-244, 1963.

WENJIAO, S.; FULU, T.; ZHAO, Z. A review on statistical models for identifying climate contributions to crop yields. Journal of Geographical Sciences, v. 23, p. 567-576, 2013.

WILKS, D. S. Statistical Methods in the Atmospheric Sciences. 2. ed. San Diego, California, United States of America: Academic Press, Elsevier, 2006. 630 p.

WMO. Guide to Agricultural Meteorological Practices. [S.1.]: World Meteorological Organization, 2012. 799 p.

YUAN, W.; GELLER, M.A.; LOVE, P.T.: ENSO influence on QBO modulations of the tropical tropopause. Quarterly Journal of the Royal Meteorological Society, p. 16701676, 2014.

ZUBAIR, L. El Niño Southern Oscillation influences on rice production in Sri Lanka. International Journal of Climatology, v. 22, p. 249-260, 2012.

License information: This is an open-access article distributed under the terms of the Creative Commons Attribution License (type CC-BY), which permits unrestricted use, distribution and reproduction in any medium, provided the original article is properly cited. 